\title{
Mischfinanzierungen in der Bundesstaatsreform
}

Mischfinanzierung bedeutet, dass eine Aufgabe durch Bund und Länder gemeinsam finanziert wird ${ }^{1}$. Auch die - grundsätzlich als Teil der Länder anzusehenden ${ }^{2}-$ Gemeinden können beteiligt sein. Mischfinanzierungen treten vor allem - wenn auch nicht nur ${ }^{3}$ - in solchen bundesstaatlichen Ordnungen auf, in denen - wie im Grundgesetz - die Kompetenzverteilung zwischen Zentral- und Gliedstaaten funktionenorientiert erfolgt, d.h. dass nicht einer Gebietskörperschaft sämtliche Staatsfunktionen (Rechtsetzung, Vollziehung, Rechtsprechung und Finanzierung) in Bezug auf eine Sachaufgabe einheitlich zugewiesen werden ${ }^{4}$. Mischfinanzierungen stellen sich ferner als Ausprägung eines bestimmten Föderalismusverständnisses dar, bei dem nicht die klare Aufgabentrennung, sondern die Kooperation der verschiedenen Ebenen des Bundesstaats im Vordergrund steht ${ }^{5}$. Solche kooperativen, die Ebenentrennung durchbrechenden Elemente in der Kompetenzstruktur waren und sind nicht auf die Finanzverfassung beschränkt. Die durch die Föderalismusreform $2006^{6}$ abgeschaffte

1 R. Borell, Mischfinanzierungen, 1981, S. 9.

2 BVerfGE 86, 148 (215); H.-G. Henneke, Öffentliches Finanzwesen, Finanzverfassung, 2. Aufl. 2000, Rdnr. 826; H. Siekmann, in: Sachs (Hrsg.), GG, 4. Aufl. 2007, vor Art. 104a Rdnr. 9 f.

3 Hauptbeispiel für ein ähnliches Problem bei andersartiger Struktur der bundesstaatlichen Kompetenzverteilung bilden die USA mit den zahlreichen Bundeshilfen an die Gliedstaaten; vgl. dazu W. Heun, Die bundesstaatliche Finanzverfassung der USA, StwStPr. 5 (1994), S. 97 ff.; ders., Die Zusammenführung der Aufgaben- und Ausgabenverantwortung von Bund, Ländern und Gemeinden als Aufgabe einer Reform der Finanzverfassung, DVB1. 1996, S. 1020 (1023).

$4 \mathrm{Zu}$ dieser Unterscheidung etwa $C$. Waldhoff, Verfassungsrechtliche Vorgaben für die Steuergesetzgebung im Vergleich Deutschland-Schweiz, 1997, S. 80 ff.; F. Ossenbühl, Föderalismus nach 40 Jahren Grundgesetz, DVB1. 1989, S. 1230 (1232); S. Korioth, Neuordnung der Bund-Länder-Finanzbeziehungen? ZG 2007, S. 1 (3 f.).

5 H. Soell, Sind die Gemeinschaftsaufgaben nach Art. 91a GG ein geeignetes Instrument zur Weiterentwicklung des föderativen Systems? in: FS für Ernst Forsthoff zum 70. Geb., 2. Aufl. 1974, S. 397 ff.; K.M. Hettlage, Mitplanung und Mitfinanzierung von Länderaufgaben durch den Bund, in: FS für Karl Carstens, Bd. 2, 1984, S. 613 (614 ff.); W. Jakob, Forschungsfinanzierung durch den Bund, Der Staat 24 (1985), S. 527 (530), spricht bei »Finanzierungszuständigkeiten aufgrund von Gemeinschaftsaufgaben i.w.S. « treffend von »staatszielakzessorischer Finanzierung «, wobei sich dies gegen die klare Kompetenztrennung richte; ferner insgesamt $B$. Tiemann, Gemeinschaftsaufgaben von Bund und Ländern in verfassungsrechtlicher Sicht, Diss. iur. München 1969; allgemein zu dem Zusammenhang zwischen »Föderalismusverständnis « und finanzverfassungsrechtlicher Argumentation K. Vogel/C. Waldhoff, in: Dolzer/Vogel/Graßhof (Hrsg.), Bonner Kommentar zum Grundgesetz, Stand: 132. Lfg. Februar 2008, Vorb. z. Art. 104a-115, Rdnr. 70 (=dies., Grundlagen des Finanzverfassungsrechts, 1999, Rdnr. 70); zum kooperativen Föderalismus im US-amerikanischen Vorbild H. Ehringhaus, Der kooperative Föderalismus in den Vereinigten Staaten von Amerika, 1971.

6 Gesetz zur Änderung des Grundgesetzes vom 28. August 2006, BGB1. I, 2034; Föderalismusreform-Begleitgesetz vom 5. September 2006, BGBl. I, 2098. 
Rahmengesetzgebungskompetenz des Art. 75 GG ermöglichte eine gemeinschaftliche gesetzliche Regelung in bestimmten Sachbereichen; die Gemeinschaftsaufgaben der Art. 91a f. GG regelten und regeln noch vor Fragen einer Mischfinanzierung eine von der Verfassung geforderte oder zumindest ermöglichte Form der Mischverwaltung ${ }^{7}$; im Bereich der dritten Gewalt besteht im Instanzenzug mit den fünf obersten Bundesgerichten als Revisionsgerichten ohnehin eine eigengeartete Verschränkung der Rechtsprechung von Ländern und Bund.

Art. 104a Abs. 1 GG stellt nach allgemeiner Ansicht ein grundsätzliches Verbot der Mischfinanzierung unter dem GG dar; Ausnahmen müssen in der Verfassung selbst zugelassen $\operatorname{sein}^{8}$. Bis zur ersten Stufe der Föderalismusreform waren dies v.a. Art. 91a Abs. 4; 91 b Satz 2 sowie Art. 104a Abs. 3 und 4 GG. Diese Bestimmungen waren Änderungen durch die Föderalismusreform $2006^{9}$ ausgesetzt. Bei der Reform von Bestimmungen der Mischfinanzierung handelt es sich demnach um den (kleinen) finanzverfassungsrechtlichen Teil der Bundesstaatsreform, der bereits verwirklicht ist ${ }^{10}$. Es wird zu prüfen sein, ob der Reformbedarf damit erledigt ist.

Die weiteren in diesem Zusammenhang relevanten Normen des Art. 106 Abs. 8 (Ausgleich bundesrechtlich veranlasster besonderer Aufgaben); 106a (Finanzierungszuschüsse zum öffentlichen Personennahverkehr) und des Art. 120 GG (Bundesfinanzierungslasten bei Kriegsfolgelasten und im Bereich der Sozialversicherung) bleiben im Folgenden außer Betracht. Auch die europaspezifischen neueingefügten Lastentragungsregeln der Art. 104a Abs. 6 und Art. 109 Abs. 5 GG in der seit dem 1. September 2006 geltenden Fassung bleiben ausgeklammert, obgleich es sich auch bei ihnen um verfassungsrechtlich positivierte Tatbestände von Mischfinanzierungen handelt. Die zuletzt genannten Bestimmungen leiden unter dem Geburtsfehler, das Pferd von hin-

7 U. Volkmann, in: Starck (Hrsg.), GG, Bd. 3, 5. Aufl. 2005, Art. 91a Rdnr. 32 sieht in der Verknüpfung ein »Wesen der Gemeinschaftsaufgaben«; ähnlich S. Luther, Die Lastenverteilung zwischen Bund und Ländern nach der Finanzreform, Diss. iur. Münster 1974, S. 105 ff., 111 ff.; Hettlage (Fn. 5), S. 623 f. Der Unterschied zwischen Art. 91a und 104a Abs. 4 GG a.F., jetzt Art. 91a n.F. und 104b GG, liegt dann darin, dass nur bei den Gemeinschaftsaufgaben Mischverwaltung und Mischfinanzierung zwingend zusammenhängen; zur Mischverwaltung im Bundesstaat allgemein und ihren Grenzen BVerfGE 63, 1 (36 ff.); BVerfG v. 20.12.2007 - »Hartz IV «, NVwZ 2008, 183 = DVBl. 2008, 173; Anmerkung $C$. Waldhoff, Die Selbstverwaltung der Kreise zwischen eigenverantwortlicher Aufgabenwahrnehmung und verfassungswidriger Mischverwaltung, ZSE 2008, S. 57; allgemein P. Lerche, in: Maunz/Dürig, GG, Art. 83 Rdnr. 85 ff.

8 BVerfGE 26, 338 (390 f.); BVerwGE 44, 351 (364); 81, 312 (314); BGH, NJW 1987, 1625 (1627); K. Stern, Das Staatsrecht der Bundesrepublik Deutschland, Bd. 2, 1980, S. 1141; $R$. Prokisch, in: Dolzer/Vogel/Graßhoff (Hrsg.), BK-GG, Art. 104a Rdnr. 116; W. Heun, in: Dreier (Hrsg.), GG, Bd. 3, 2000, Art. 104a Rdnr. 17; Siekmann (Fn. 2), Art. 104a Rdnr. 12; sog. ungeschriebene Finanzierungszuständigkeiten des Bundes sind allenfalls noch aufgrund der - restriktiv zu handhabenden - ungeschriebenen Verwaltungszuständigkeiten des Bundes möglich, keinesfalls darüber hinaus, vgl. m.w.N. J. Hellermann, in: Starck (Hrsg.), GG, Bd. 3, 5. Aufl. 2005, Art. 104a Rdnr. 141 ff. insbes. 149.

9 Siehe oben Fn. 6.

$10 \mathrm{Zu}$ diesen finanzverfassungsrechtlichen Änderungen insgesamt Hellermann, in: Starck (Hrsg.), Föderalismusreform, 2007, Rdnr. 272 ff. 
ten aufgezäumt zu haben: Ohne eine Regelung der primären und damit wichtigeren Frage einer vertikalen und horizontalen Aufteilung der Verschuldungsanteile zwischen Bund, Ländern, Kommunen und sonstigen juristischen Personen des öffentlichen Rechts ist die Neuregelung als »[k]onzeptionslos, unvollständig, mit Detailfehlern und absehbaren Ungereimtheiten in der Anwendung belastet « charakterisiert worden ${ }^{11}$.

Der wohl spektakulärste Fall einer Mischfinanzierung in jüngerer Zeit stellt die teilweise vor dem Bundesverfassungsgericht gescheiterte sog. Hartz IV-Reform (Einführung einer sog. Grundsicherung für Arbeitslose nach dem SGB II) dar. Stefan Korioth hat darauf hingewiesen, dass diese die Gesamtsumme aller bisherigen Mischfinanzierungen um das Sechsfache (!) übersteigende Mischfinanzierung bemerkenswerter Weise in der finanzverfassungsrechtlichen Reformdiskussion bisher unterbelichtet geblieben sei $^{12}$. Die beschwerdeführenden Kreise hatten mit ihrer Kommunalverfassungsbeschwerde die Verfassungswidrigkeit von $§ 46$ Abs. 1 und Abs. 5 bis 10 SGB II gerügt, weil diese Norm ohne Einschaltung der Länder durch die verbindliche Festlegung, welche Ausgleichsleistungen die kommunalen Träger der Grundsicherung aus dem Bundeshaushalt erhalten, in die verfassungsrechtlich geschützte kommunale Finanzhoheit eingreife. Dem lag die Überlegung zugrunde, dass direkte Finanzbeziehungen zwischen Bund und Kommunen in der grundsätzlich zweistufigen Finanzverfassung jenseits des Ausnahmefalls des Art. 106 Abs. 8 GG unzulässig sind ${ }^{13}$. Das Gericht interpretiert die angegriffene Norm demgegenüber dahingehend, dass lediglich die Länder einen Anspruch gegen den Bund erwürben und dann nach Landesrecht verpflichtet seien, die Geldmittel an die Kreise weiterzureichen ${ }^{14}$. Allein die in Abs. 5 der Vorschrift deutlich werdende Intention, die Kommunen durch die Zahlungen des Bundes finanziell zu entlasten, führe nicht zu einer direkten Rechtsbeziehung zwischen Bund und Kreisebene, sodass eine Verletzung von Art. 84 Abs. 1 GG a.F. und Art. 28 Abs. 2 GG ausscheide. Auf diesen Sonderfall wird im Folgenden - unabhängig von der Überzeugungskraft der verfassungsgerichtlichen Argumentation - ebenfalls nicht eingegangen ${ }^{15}$.

Im Folgenden sollen zunächst Entstehung und Entwicklung der Mischfinanzierungstatbestände unter dem Grundgesetz nachgezeichnet werden (unter I.), bevor die Änderungen des Jahres $2006 \mathrm{zu}$ analysieren sind (unter II.). Anschließend ist die Mischfinanzierung als Mehrebenenproblem darzustellen (unter III.), um dann auf die grundsätzliche Problematik der bundesstaatlichen Verflechtung einzugehen (unter IV.). Abschließend werden Schlussfolgerungen für die anstehenden und auch vom Bundesverfassungsgericht inzwischen ausdrücklich eingeforderten ${ }^{16}$ Reformschritte

11 Korioth (Fn. 4), S. 7; ausführlich jetzt K. Knop, Verschuldung im Mehrebenensystem, 2008, (im Erscheinen).

12 (Fn. 4), S. 6.

13 BVerfGE 26, 172 - 186, hier: Leitsatz 1: »Der Bund ist weder berechtigt noch verpflichtet, die finanziellen Verhältnisse der Gemeinden unmittelbar ohne Einschaltung der Länder zu ordnen. ...«; C. Waldhoff, Grundzüge des Finanzrechts des Grundgesetzes, in: Isensee, J./ Kirchhof, P. (Hg.), HStR V, 3. Aufl. 2007, § 116 Rdnr. 17 f.

14 Tz. $140 \mathrm{ff}$.

15 Vgl. die Urteilsanmerkung Waldhoff (Fn. 7), S. 57.

16 BVerfGE 116, 327 (389 f.) - »Berlin-Urteil«. 
durch die mit Beschlüssen vom 15. Dezember 2006 eingesetzte »Gemeinsame Kommission zur Modernisierung der Bund-Länder-Finanzbeziehungen $«{ }^{17}$ im Hinblick auf Mischfinanzierungstatbestände gezogen (unter V.).

\section{Die Entwicklung der Mischfinanzierung bis zur Föderalismusreform}

Der 1969 eingefügte Art. 104a GG stellt die erste ausdrückliche Regelung einer föderalen Finanzierungskompetenz in der deutschen Verfassungsgeschichte dar ${ }^{18}$. Finanzierungskompetenz wird hier als Oberbegriff für Finanzierungslast und Finanzierungsbefugnis verwendet: Finanzierungslast (Ausgabenlast; Finanzaufgabe) meint die Pflicht, bestimmte Ausgaben zu tragen; Finanzierungsbefugnis thematisiert das Problem, ob eine Ebene Mittel für eine bestimmte Aufgabe bereitstellen darf ${ }^{19}$. Zumeist fallen beide Kategorien zusammen, müssen dies jedoch nicht ${ }^{20}$.

Die Reichsverfassung von 1871, die WRV, wie auch das GG bis zur Finanzverfassungsreform $1955^{21}$, gingen implizit vom »Grundsatz der synchronisierten Aufgabenund Finanzverteilung « ${ }^{22}$ aus. Das Finanzverfassungsgesetz $1955^{23}$ sprach in dem neu gestalteten und bis 1969 geltenden Art. 106 Abs. 4 Satz 2 Nr. 1 davon, dass »Bund und Länder ... gesondert die Ausgaben, die sich aus der Wahrnehmung ihrer Aufgaben ergeben « »tragen «. Diese ohnehin in anderem Zusammenhang (der Revision der Verteilung des Aufkommens von Einkommen- und Körperschaftsteuer zwischen Bund und Ländern) getroffene Regel wurde zwar als verallgemeinerungsfähig anerkannt ${ }^{24}$, konnte die Ausbreitung der um sich greifenden sog. Fondsverwaltung ${ }^{25}$, d.h. der Dotation bestimmter Aufgaben und Projekte der Länder durch Bundesmittel, nicht steu$\mathrm{ern}^{26}$. Die Landeskompetenzen waren dadurch im Kern berührt. Die Hingabe von Finanzmitteln impliziert - gemäß der Volksweisheit »wer zahlt, schafft an« - als

17 BT-Drs. 16/3885 und BR-Drs. 913/06; zu den damit verbundenen Reformaufgaben insgesamt Korioth (Fn. 4); P. Selmer, Zur Reform der bundesstaatlichen Finanzverfassung, NVwZ 2007, S. 872; R. Wendt, Neuordnung der Finanzbeziehungen im Bundesstaat, in: FS Jung, 2007, S. 1085.

18 I. Kesper, Bundesstaatliche Finanzordnung, 1998, S. 74; J.-P. Schneider, in: AK-GG, 3. Aufl. 2001 ff., Art. 104a Rdnr. 1.

19 H.H. von Arnim, Finanzzuständigkeit, in: Isensee/Kirchhof (Hrsg.), HStR IV, 2. Aufl. 1999, $\S 103$ Rdnr. 10; Heun (Fn. 8), Art. 104a Rdnr. 17.

20 Korioth (Fn. 4), S. 10 Fn. 20.

21 Zur Entwicklung der Finanzverfassung von 1949 bis 1955 m.w.N. Vogel/Waldhoff (Fn. 5), Rdnr. 199 ff.

22 Friedrich Klein, Von der föderativen zur stärker unitarischen Gestaltung des Finanzwesens in der Bundesrepublik Deutschland, in: FS Giese, 1953, S. 61 (90).

23 Vom 23. Dezember 1955, BGBl. I, 817.

24 BVerfGE 26, 338 (389 f.): »Der Vorschrift kommt ... die Bedeutung einer allgemeinen, das Bund/Länder-Verhältnis im ganzen bestimmenden Lastenverteilungsregel zu. ... Der am 1. Januar 1970 in Kraft tretende Art. 104a Abs. 1 GG ... enthält insofern nur eine Klarstellung $\ldots \ll$

25 Vgl. die Analysen von A. Köttgen, Fondsverwaltung in der Bundesrepublik, 1965 und G. Kisker, Kooperation im Bundesstaat, 1971, S. 34 ff.

26 Heun (Fn. 8), Art. 91a Rdnr. 2. 
»Angebotsdiktatur« stets auch wesentliche inhaltliche Einflussnahme auf die Politik des Empfängers. Zudem wurden durch mischfinanzierte Projekte zusätzliche Haushaltsmittel der Länder gebunden, die für autonome Landespolitik nicht mehr zur Verfügung standen: »Die Unterfinanzierung der finanzschwachen Länder führte zu wachsenden Abhängigkeiten vom Bund, und in der Folge zu einem auch für Experten kaum mehr überschaubaren Komplex von Mischfinanzierungen, durch die der Bund in die Aufgabenbereiche der Länder eindrang. Faktisch entwickelte sich damit abseits der Zuständigkeitsregelung des Grundgesetzes eine >Grauzone $<$, die von nahezu allen Beteiligten als kritikwürdig empfunden wurde. $\ll^{27}$ Die in zwei Stufen durchgeführte sog. Große Finanzreform 1967/69 stellte die Antwort auf die geschilderte Situation durch den Versuch einer Verrechtlichung des parakonstitutionellen Zustands dar ${ }^{28}$. Ausgehend von einem klaren Konzept - dem sog. kooperativen Föderalismus - sollte unter Zugrundelegung des Leitbilds des »unitarischen Bundesstaats $~^{29}$ und vor dem Hintergrund eines sozialtechnokratischen Politikverständnisses der Plan- und Machbarkeit, verbunden mit einem keynesianischen ökonomischen Ansatz die bundesstaatliche Ordnung und vor allem die bundesstaatliche Finanzverfassung den Anforderungen des international verflochtenen modernen Sozialstaats, d.h. den »Anforderungen der Zeit «, angepasst werden ${ }^{30}$. Stefan Oeter hat den Hintergrund des Gutachtens und der Reform treffend charakterisiert: »Die theoretischen Prämissen dieses Konzeptes aber waren nicht weniger Produkte der Zeit als die Interessenlagen der letztlich den Kompromiss bestimmenden politischen Akteure. Das von Tradition aus unitarische Denken der intellektuellen und administrativen Eliten Deutschlands ging hier ein Bündnis ein mit den intellektuellen Moden der Zeit, der Planungs- und Verflechtungseuphorie der späten Sechziger Jahre, unter dem Einfluss auch bestimmter Vorbilder des verbündeten Auslands, wie >planification $<$ und $>$ cooperative federalism $<. \ll{ }^{31}$ Die Krönung der möglichst flexibel zu gestaltenden, neuen Zusammenarbeitsformen von Bund und Ländern sollten die Gemeinschaftsaufgaben sein. Wurde von der Troeger-Kommission noch eine durch Bundesgesetz auszufüllende Generalklausel vorgeschlagen, setzte sich in den Gesetzesberatungen in deutlicher Abschwächung dieses Entwurfs ein abschließender Katalog für die einer gemeinsamen Wahrnehmung geeigneten Sachmaterien in Form der bis September 2006 geltenden Art. 91a, b GG a.F. durch. Die Fondsverwal-

27 S. Oeter, Integration und Subsidiarität im deutschen Bundesstaat, 1998, S. 275.

28 Vgl. die Darstellung bei I. von Münch, Gemeinschaftsaufgaben im Bundesstaat, VVDStRL 31 (1973), S. 51 (52 ff.); W. Renzsch, Finanzverfassung und Finanzausgleich, 1991, S. 209 ff.; Vogel/Waldhoff (Fn. 5), Rdnr. 211 ff.

29 K. Hesse, Der unitarische Bundesstaat, 1962, mit der einseitigen Betonung der Funktion der vertikalen Gewaltenteilung.

30 Die Reform wurde durch das sog. Troeger-Gutachten vorbereitet: Kommission für die Finanzreform, Gutachten über die Finanzreform in der Bundesrepublik Deutschland, 2. Aufl. 1966; gute Darstellung und Charakterisierung bei Oeter (Fn. 27), S. 274 ff.

31 (Fn. 27), S. 290; zur traditionell zentralistischen Grundprägung vieler Akteure und Diskussion der Finanzverfassung und ihren Hintergründen in je unterschiedlichen Zusammenhängen Waldhoff, Verfassungsrechtliche Vorgaben (Fn. 4), S. 81 ff.; ders., Reformperspektiven im Finanzrecht, Die Verwaltung 39 (2006), S. 155 (167 ff.); ders., Grundzüge (Fn. 13), Rdnr. 79 . 
tung wurde durch Art. 104a Abs. 1 GG verfassungsrechtlich eingebunden. Zugleich wurden jedoch - neben den Gemeinschaftsaufgaben - in den Absätzen 3 und 4 explizite Durchbrechungen normiert. Bei den Gemeinschaftsaufgaben muss das Element der Mischfinanzierung - notwendig verbunden mit der gemeinsamen Rahmenplanung als Form zulässiger Mischverwaltung - als das Hauptmotiv angesehen werden ${ }^{32}$. Die Rechtsprechung des Bundesverfassungsgerichts hatte schon bald Gelegenheit die ärgsten Übertreibungen im Zusammenhang mit den Neuregelungen am Beispiel der Investitionshilfekompetenz des Art. 104 Abs. 4 GG a.F. einzuschränken ${ }^{33}$. In den Urteilen zum Städtebauförderungsgesetz ${ }^{34}$ und zur Strukturförderung ${ }^{35}$ war das Gericht der nahe liegenden Tendenz entgegengetreten, Art. 104a Abs. 4 GG a.F. als Legitimation einer pauschalen Fortführung der überkommenen Dotationspraxis des Bundes zu sehen und hatte den Ausnahmecharakter der Vorschrift betont. Trotz dieser Ansätze und trotz des Haushaltsvorbehalts in Art. 91a Abs. 4 Satz 4 GG a.F. konnte die »Angebotsdiktatur « des Bundes - seine berühmten »goldenen Zügel«, an denen er die Länder führte mit den Folgen einer weitgehenden Entmachtung der Landesparlamente aufgrund der exekutiv ausgehandelten, zu weitreichenden faktischen Bindungen führenden Projekte - kaum aufgehalten werden. In den Worten Fritz Ossenbühls: »Kooperativer Föderalismus ist Exekutivföderalismus, weil die Entscheidungsergebnisse durch administrative Gremien ausgehandelt und faktisch festgelegt werden. ... Damit ist das parlamentarische Entscheidungssystem als Ganzes berührt. Der kooperative Föderalismus führt als Exekutivföderalismus zum >oligarchischen Bundesstaat< (Gunter Kis$k e r) .{ }^{36}$. In der Tendenz erwiesen und erweisen sich Mischfinanzierungen als ausgabensteigernd, nicht als ausgabenbegrenzend ${ }^{37}$. Die in den Gemeinschaftsaufgaben und in den sonstigen Mischfinanzierungstatbeständen angestrebte Politikverflechtung hat in die »Politikverflechtungsfalle« (Fritz.W. Scharpf), d.h. letztlich in eine moderne Form obrigkeitsstaatlicher Exekutivherrschaft ohne für den Bürger sichtbar zurechenbare oder durchschaubare Verantwortlichkeiten mit entsprechenden Auswirkungen auf die demokratische Sanktion des Wählers geführt ${ }^{38}$.

$32 \operatorname{Heun}$ (Fn. 8), Art. 91a Rdnr. 32.

$33 \mathrm{Zu}$ den letztlich wirkungslos gebliebenen Einschränkungsversuchen in der Literatur vgl. die Zusammenstellung bei von Arnim (Fn. 19), Rdnr. 49 ff.

34 BVerfGE 39, 96 (107 ff.).

35 BVerfGE 41, 291 (304 ff.).

36 (Fn. 4), S. 1235; ferner R. Wendt, Finanzhoheit und Finanzausgleich, in: Isensee/Kirchhof (Hrsg.), HStR IV, 2. Aufl. 1999, § 104 Rdnr. 93 ff.; Heun (Fn. 8), Art. 91a Rdnr. 8, 34 f.; treffende Gefahrendiagnose auch in BVerfGE 39, 96 (107); gute Diagnose bezogen auf ein bestimmtes Feld auch bei C. Gramm, Bewährungsprobe für die Gemeinschaftsaufgabe Hochschulbau, WissR 26 (1993), S. 198 (207 ff.); eines der letzten prominenten Beispiele unter der Rechtslage vor der Föderalismusreform war das sog. Ganztagsschulprogramm des Bundes; dazu jeweils kritisch R. Stettner, Der verkaufte Verfassungsstaat, ZG 2003, S. 315 (321 ff.); C. Winterhoff, Finanzielle Förderung von Ganztagsschulen und Juniorprofessuren durch den Bund?, JZ 2005, S. 59 (62 f.); U. Häde, Zur Föderalismusreform in Deutschland, JZ 2006, S. 930 (936).

37 Borell (Fn. 1), S. 42 ff.

38 F. W. Scharpf/B. Reissert/F. Schnabel, Politikverflechtung, 1976; F.W. Scharpf, Optionen des Föderalismus in Deutschland und Europa, 1994; Wendt, Finanzhoheit (Fn. 36), Rdnr. 93; 
Die vom Bundesverfassungsgericht benannte Funktion von Mischfinanzierungen als Handlungsinstrument zur Bewältigung von Haushaltsnotlagen ${ }^{39}$ ist bisher kaum entfaltet worden.

\section{Die Änderungen der Mischfinanzierungstatbestände durch die Föderalismusre- form}

Es gehörte zur ausdrücklichen Agenda der Föderalismuskommission, »die Finanzbeziehungen (insbesondere Gemeinschaftsaufgaben und Mischfinanzierung) zwischen Bund und Ländern [zu] überprüfen ${ }^{40}{ }^{0}$, galten Gemeinschaftsaufgaben und Mischfinanzierung doch als Hort bundesstaatlicher Verflechtung, als »geradezu prototypisch für das Konzept eines kooperativen Föderalismus«. Sie standen damit unter dem Generalverdacht, »der Verantwortungsvermischung und Ineffizienz Vorschub zu leisten $\aleph^{41}$. Gleichwohl wurden Gemeinschaftsaufgaben beibehalten. Sie wurden allerdings »modernisiert « und »entbürokratisiert ${ }^{42}$ : In Art. 91a Abs. 1 GG n.F. wurde die alte Ziffer 1, den Hochschulbau betreffend, gestrichen und verändert in Art. 91b GG n.F. aufgenommen, aus den echten Gemeinschaftsaufgaben somit entfernt. Die Regelung der Ausgabentragung ist von Abs. 4 in Abs. 3 des Art. 91a vorgezogen, in der Sache jedoch unverändert geblieben. Das Zurückdrängen des Hochschulbaus erklärt sich aus der Beendigung der Expansion der Hochschulen. Die Finanzierungsprobleme liegen z.Z. eher in der Unterhaltung der gebauten Hochschulen und ihrer Gebäude, als in der Notwendigkeit von Neubauten ${ }^{43}$. Größere Änderungen haben sich im Rahmen der

Kesper (Fn. 18), S. 218 ff.; zur Entmachtung der Landesparlamente m.w.N. J.A. Frowein, Gemeinschaftsaufgaben im Bundesstaat, VVDStRL 31 (1973), S. 13 (24 ff.); die EnqueteKommission Verfassungsreform hat sich nach einiger Diskussion für die Beibehaltung von Gemeinschaftsaufgaben und Mischfinanzierung bei einer inhaltlichen Straffung ausgesprochen, Beratungen und Empfehlungen zur Verfassungsreform II, Zur Sache 2/77, S. 180 f.; vgl. dazu J. Müller-Volbehr, Gemeinschaftsaufgaben und Investitionshilfe, DVBl. 1978, S. 313.

39 BVerfGE 86, 148 (265); 116, 327 (389 f.); dazu Wendt (Fn. 17), S. 1104 ff., der in seiner Argumentation jedoch zu wenig berücksichtigt, dass auch bei der zwingend vorgeschriebenen gleichen Berücksichtigung der geförderten Länder schon durch die unterschiedlichen tatsächlichen Ausgangslagen »Umverteilungseffekte« eintreten; wie hier Heun (Fn. 8), Art. 91a Rdnr. 33; differenziert Prokisch (Fn. 8), Rdnr. 280 ff.

40 BT-Drs. 15/1685; vgl. näher $O$. Runde, Finanzen: Gemeinschaftsaufgaben/Mischfinanzierungen, in: Holtschneider/Schön (Hrsg.), Die Reform des Bundesstaates, 2007, S. 297 ff.; $J$. Riebel, Reduzierung von Mischfinanzierungen, ebd., S. $320 \mathrm{ff}$.

41 Hellermann (Fn. 10), Rdnr. 276; E. Schmidt-Jortzig, Die fehlgeschlagene Verfassungsreform, ZG 2005, S. 16 (26): Art. 91a GG a.F. als »Hort an Intransparenz, bürokratischem Aufwand, ökonomischer Unwirksamkeit und rechnungsprüferischer Resistenz«; ferner D. Merten, Die Reform der funktionalen Kompetenzverteilung im Bund-Länder-Verhältnis, in: Blanke/Schwanengel (Hrsg.), Zustand und Perspektiven des deutschen Bundesstaates, 2005, S. 79 (85).

42 Hellermann (Fn. 10), Rdnr. 279, 285.

43 Hellermann (Fn. 10), Rdnr. 283; kritisch S. Westerburg, Auswirkungen der Föderalismusreform auf die rechtlichen Rahmenbedingungen von Wissenschaft und Forschung, WissR 39 (2006), S. 338 (342 f.); Korioth (Fn. 4), S. 5 f.; zur Hochschulbaufinanzierung unter der alten Rechtslage umfassend Gramm (Fn. 36). 
unechten Gemeinschaftsaufgaben bei Art. 91b GG ergeben: »Bildungsplanung « sowie »Förderung von Einrichtungen und Vorhaben der wissenschaftlichen Forschung von überregionaler Bedeutung « in der alten Fassung sind ersetzt worden durch einen dreiziffrigen Katalog der betroffenen Materien: »1. Einrichtungen und Vorhaben der wissenschaftlichen Forschung außerhalb von Hochschulen; 2. Vorhaben der Wissenschaft und Forschung an Hochschulen; 3. Forschungsbauten an Hochschulen einschließlich Großgeräten « in der neuen Fassung. Im Bereich der nunmehr in Abs. 3 geregelten Regelung zur »Kostentragung « tritt dieser Begriff an den ursprünglich verwendeten Passus »Aufteilung der Kosten«, der nach überwiegender Meinung die jetzt ermöglichte Alleinfinanzierung durch den Bund versperrte ${ }^{44}$. Die finanzielle Entlastung der Länder wird hier mit einer Steigerung des Einflusses des Bundes erkauft ${ }^{45}$. Weiter ungeklärt bleibt die Streitfrage, ob im Rahmen der Förderziele des Art. 91b GG über die Zweckausgaben auch Verwaltungsausgaben erfasst werden können.

Auch im Hinblick auf Art. 104a GG wurde die Grundkonzeption nicht verändert. Der die verfassungspolitische Diskussion bisher stark bestimmende Bundesdurchgriff auf die Kommunen ist auch und gerade hinsichtlich der Finanzierungslast durch Art. 84 Abs. 1 Satz 7; 85 Abs. 1 Satz 2 GG n.F. entschärft worden ${ }^{46}$. Entsprechendes gilt für die - dem Postulat der Entflechtung allerdings zuwiderlaufende - neugeschaffene Zustimmungspflicht des Bundesrats bei Geldleistungsgesetzen, an deren Kosten die Länder beteiligt sind (Art. 104a Abs. 4 GG n.F.). Damit war auch der Druck zum Übergang von der Verwaltungsanknüpfung zur Gesetzeskausalität im Rahmen des Konnexitätsprinzips zumindest vermindert. Die offene Flanke der Finanzhilfen des Bundes wurde durch den neueingefügten Art. 104b, der an die Stelle des alten Art. 104a Abs. 4 GG tritt, vermindert: Diese dürfen nur noch befristet und degressiv gestaffelt und nur in Bereichen, in denen dem Bund eine Gesetzgebungskompetenz zusteht ${ }^{47}$, geleistet werden. Durch den Bund initiierte Schulversuche oder sein $\gg$ Ganztagsschulprogramm $\aleph^{48}$ wären mangels Bundeszuständigkeit im Schulbereich damit nicht mehr zulässig ${ }^{49}$.

Das Kompromisshafte der Veränderungen bei den Mischfinanzierungen wird besonders in den langfristigen, nicht unproblematischen Übergangsregelungen (Art. 143c GG $)^{50}$ sowie im ausführenden sog. Entflechtungsgesetz deutlich ${ }^{51}$.

44 B. Pieroth, in: Jarass/Pieroth, GG, 9. Aufl. 2007, Art. 91b Rdnr. 6.

45 Vgl. I. Kesper, Reform des Föderalismus in der Bundesrepublik Deutschland, NdsVBl. 2006, S. 145 (152); Hellermann (Fn. 10), Rdnr. 310.

46 F. Eckardt/D. Buscher, Föderalismusreform II: Reform der Finanzbeziehungen von Bund, Ländern und Kommunen, DÖV 2007, S. 89 (91 f.); Hellermann (Fn. 10), Rdnr. 316.

47 Ausgeschlossen ist nach Art. 104b Abs. 1 GG die Förderung im Bereich ausschließlicher Gesetzgebungszuständigkeit der Länder; unklar ist, ob im Bereich der konkurrierenden Zuständigkeit die Erforderlichkeitsklausel des Art. 72 Abs. 2 GG greift; dies dürfte zu bejahen sein, wie die Formulierung »soweit dieses Grundgesetz ihm Gesetzgebungsbefugnisse verleiht « nahelegt; a.A. Kesper (Fn. 45), S. 152 f. mit Fn. 60.

48 Siehe die Nachweise oben Fn. 36.

49 K.-M. Reineck, Die Föderalismusreform 2006, DVP 2006, S. 485 (490); Kesper (Fn. 45), S. 152.

50 Vgl. nur Kesper (Fn. 45), S. 153; K.-A. Schwarz, in: Starck (Hrsg.), Föderalismusreform, 2007, Rdnr. 142 ff., $413 \mathrm{ff}$.

51 Art. 13 des Föderalismusreformbegleitgesetzes, s.o. Fn. 6. 
Die finanzverfassungsrechtlichen Neuregelungen und darin die Veränderung der Mischfinanzierungstatbestände durch die Föderalismusreform I sind in der Literatur ganz überwiegend auf Kritik gestoßen: Die Reform habe stets unter das Fehlen einer Grundsatzdiskussion hervorgerufener Konzeptionslosigkeit gelitten, sie sei von Anfang an - neben »Verlegensheitsformeln« wie »Modernisierung « oder »Anpassung an geänderte Rahmenbedingungen « - durch teilweise widersprüchliche Vorgaben geprägt gewesen, die sich in ambivalenten Signalen von Entflechtung und erneuter Verflechtung geäußert hätten ${ }^{52}$. Im Hinblick auf die Mischfinanzierung wird das Ergebnis angesichts des ursprünglichen Vorhabens einer Abschaffung der Gemeinschaftsaufgaben als »ernüchternd « eingestuft ${ }^{53}$, die begrenzte, bloß modifizierende Neuordnung habe sich »weder zu einem klaren Ja oder Nein der Mischfinanzierungen ... durchringen können «: »So bleibt es bei den seit langem kritisierten Wirkungen der Mischfinanzierungen, ausgabensteigernden Effekten durch hohen Koordinierungs- und Verwaltungsaufwand sowie Mitnahmeeffekten infolge angebotener Bundesmittel, die eine ländereigene Präferenzbildung verzerren. ${ }^{54}$ Die Abschaffung der Gemeinschaftsaufgaben wäre die bessere Lösung gewesen ${ }^{55}$.

\section{Die europarechtliche Dimension der Mischfinanzierungsproblematik}

Finanzverfassungsrechtliche (Reform-)Fragen können heute nur noch in der Mehrebenendimension erörtert werden ${ }^{56}$. Die ökonomische wie rechtliche Interdependenz zu anderen Staaten wie zu supranationalen Verbünden kommt gerade im Finanzbereich deutlich zum Ausdruck. Das gilt auch für die Ausgabenkompetenzen und damit für die Mischfinanzierung. Im Folgenden ist die Perspektive daher um die Einbeziehung von EU bzw. EG, deren Finanzierung untrennbar mit den mitgliedstaatlichen Finanzordnungen verbunden ist ${ }^{57}$, zu erweitern. Die grundsätzliche Eigentümlichkeit der Finanzordnung der EG besteht darin, dass diese trotz des Euphemismus »Eigenmittelsystem « in Art. 269 EGV letztlich nicht über ihre Einnahmen entscheidet, diese Entscheidungen sich vielmehr die Mitgliedstaaten als »Herren der Verträge« durch die dem primären

52 Betont kritisch Korioth (Fn. 4) und Selmer (Fn. 17); milder Hellermann (Fn. 10), Rdnr. 283.

53 Selmer (Fn. 17), S. 874; ähnlich G. Färber/N. Otter, Reform der Finanzverfassung - eine vertane Chance? APuZ 13-14/2005, S. 33 (35); Kesper (Fn. 45), S. 153; I. Kemmler, Arbeit und Ergebnisse der Föderalismuskommission im Bereich der Finanzbeziehungen zwischen Bund und Ländern, in: Föderalismusjahrbuch 2005, S. 118 ff.

54 Korioth (Fn. 4), S. 5; vgl. bereits Schmidt-Jortzig (Fn. 41), S. 26 f.

55 P. M. Huber, Das Bund-Länder-Verhältnis de constitutione ferenda, in: Blanke/Schwanengel (Hrsg.), Zustand und Perspektiven des deutschen Bundesstaates, 2005, S. 21 (39 f.).

56 C. Waldhoff, Finanzautonomie und Finanzverflechtung in gestuften Rechtsordnungen, VVDStRL 66 (2007), S. 216 (219 ff.). Allgemein für Kompetenzfragen R. Breuer, Bundesstaatliche Kompetenzverordnung im europäischen Staatenverband, in: FS für Christian Starck, 2007, S. 165 (168).

57 A. Bleckmann/S. Hölscheidt, Gedanken zur Finanzierung der EG, DÖV 1990, S. 853; zur EG-Finanzierung allgemein Bettina Meermagen, Beitrags- und Eigenmittelsystem, 2002; C. Waldhoff, in: Calliess/Ruffert (Hrsg.), EUV-/EGV, 3. Aufl. 2007, Art. 268 f. EGV. 
Gemeinschaftsrecht zugehörigen Eigenmittelbeschlüsse ${ }^{58}$ vorbehalten haben. Auf der Ausgabenseite hingegen ist die Verwendung der Finanzmittel grundsätzlich vergemeinschaftet und es hat sich - bei allen Defiziten im Detail - ein spezifisch geartetes Budgetrecht des Parlaments herausgebildet ${ }^{59}$. Im Folgenden geht es um die Abgrenzung der Finanzierungszuständigkeit zwischen Gemeinschaft und Mitgliedstaaten und dabei auftretende Mischfinanzierungen, nicht um die innerbundesstaatliche Finanzierungslastverteilung beim mitgliedstaatlichen Vollzug von Gemeinschaftsrecht ${ }^{60}$.

Bei der Analyse von Mischfinanzierungen zwischen Gemeinschaft und Mitgliedstaaten ist - ganz ähnlich wie nach deutscher Finanzverfassung - zwischen Verwaltungsausgaben und operativen Ausgaben zu unterscheiden ${ }^{61}$. Sofern die Gemeinschaft ausnahmsweise durch eigene Behörden tätig wird, folgt die Finanzierungszuständigkeit für die dadurch verursachten Verwaltungsausgaben der Verwaltungszuständigkeit. Im Regelfall des mitgliedstaatlichen Verwaltungsvollzugs ${ }^{62}$ verbleiben die Verwaltungsausgaben bei den vollziehenden Mitgliedstaaten. Dieser Grundsatz wird bei der mitgliedstaatlichen Erhebung der Eigenmittel durchbrochen, wenn die Mitgliedstaaten hier pauschal $10 \%$ für ihren Verwaltungsaufwand einbehalten ${ }^{63}$. Fragen der Mischfinanzierung stellen sich in erster Linie bei den operativen Ausgaben.

Im Gemeinschaftsrecht gibt es seit Jahren eine »Tendenz zur Mischfanzierung « von Projekten zwischen Gemeinschaft und Mitgliedstaaten, insbesondere in der Agrarstrukturpolitik sowie in der Strukturpolitik allgemein ${ }^{64}$. Das Stichwort lautet: »Ko-Finanzierung «. Dieser Sachverhalt wird unter den Schlagworten der Kooperation und der Komplementarität der Förderung diskutiert ${ }^{65}$. Gemeinschaftsrechtlich existiert kein Art. 104a Abs. 1 GG vergleichbares Konnexitätsprinzip bei der Verteilung der

58 Zur Zeit gültig. Beschluss des Rates vom 7. Juni 2007 über das System der Eigenmittel der Europäischen Gemeinschaft (2007/436/EG, Euratom).

59 M. Rossi, Europäisches Parlament und Haushaltsverfassungsrecht, 1997.

$60 \mathrm{Zu}$ letzterem Heun (Fn. 8), Art. 104a Rdnr. 5; M. Heintzen, in: von Münch/Kunig (Hrsg.), GG, Bd. 3, 5. Aufl. 2003, Art. 104a Rdnr. 34; für den verfassungsrechtlich ungeregelten Fall, das das Gemeinschaftsrecht Geldleistungsansprüche - etwa für Landwirte - gewährt und die Gemeinschaft diese Kosten nicht erstattet S. Magiera, Bundesstaat und EG-Finanzordnung, in: FS Menzel, 1976, S. 621 ff.; P. Selmer, Zur bundesstaatlichen Lastenverteilung bei der Anwendung von Geldleistungsvorschriften der Europäischen Gemeinschaft, in: GS Sasse, 1981, S. 229 ff.; von Arnim (Fn. 19), Rdnr. 39 f.; U. Mager, von Münch/ Kunig (Hrsg.), GG, Bd. 3, 5. Aufl. 2003, Art. 91 Rdnr. 29 f.

61 Zur deutschen Unterscheidung zwischen Verwaltungs- und Zweckausgaben statt aller nur Henneke (Fn. 2), Rdnr. 195 f.

62 R. Streinz, Der Vollzug des Europäischen Gemeinschaftsrechts durch deutsche Staatsorgane, in: Isensee/Kirchhof (Hrsg.), HStR VII, 1992, § 182 Rdnr. 4 und durchgehend.

63 U. Häde, Finanzausgleich, 1996, S. 404 f.

64 Häde (Fn. 63), S. 418; M. Rodi, Die Subventionsrechtsordnung, 2000, S. 192, 295 f.; vgl. insgesamt M. Seidel, Subventionshoheit und Finanzierungslast in der Europäischen Wirtschaftsgemeinschaft, in: FS für Karl Carstens, Bd. 1, 1984, S. 273 ff.; A. Glaesner, Der Grundsatz des wirtschaftlichen und sozialen Zusammenhalts im Recht der Europäischen Wirtschaftsgemeinschaft, 1990, S. 29 ff.; B. Schöndorf-Haubold, Die Strukturfonds der Europäischen Gemeinschaft, 2005.

65 Heun (Fn. 8), Art. 91 Rdnr. 3 m.w.N.; ferner A. Poth-Mögele, Das Prinzip der »Partnerschaft« in der Strukturpolitik der Europäischen Gemeinschaft, 1993. 
Ausgabenkompetenzen. Daher ist auf den Grundsatz der begrenzten Einzelermächtigung zurückzugreifen, Art. 5 Abs. 1 EGV. Der EGV teilt im Regelfall der Gemeinschaft Zuständigkeiten nach Sachaufgaben, nicht nach Funktionen $\mathrm{zu}^{66}$. Die Aufgabenzuweisung umfasst somit grundsätzlich auch die Finanzierungskompetenz. Das gilt insbesondere dann, wenn der Vertrag Begriffe wie »fördern « o.ä. verwendet. Verdeutlicht werden kann dies am Beispiel der vergemeinschafteten Agrarpolitik, die immer noch den größten Ausgabenposten des Gemeinschaftshaushalts darstellt. Die Regelungen des Agrarmarkts sind europarechtlich determiniert, der Vollzug liegt im Wesentlichen bei den Mitgliedstaaten. Schon aus dieser Aufteilung folgt, dass Mitfinanzierungsbefugnisse der Gemeinschaft nicht ausgeschlossen sein können; andernfalls würde die Gemeinschaft Politiken verwirklichen, für welche die Mitgliedstaaten - zusätzlich zu ihrem Finanzierungsanteil an der Gemeinschaft - die Sachausgaben tragen müssten. In der Tat wird die Finanzierung der gemeinsamen Agrarpolitik nicht den Mitgliedstaaten überlassen, sondern durch den Europäischen Ausrichtungs- und Garantiefonds für Landwirtschaft (EAGFL) »getragen von dem Gedanken der finanziellen Solidarität der EG « übernommen ${ }^{67}$. Auch die Strukturpolitik jenseits des Agrarbereichs könnte angeführt werden. In beiden Fällen haben die grundgesetzlichen Gemeinschaftsaufgaben der Verbesserung der regionalen Wirtschaftsstruktur wie der Agrarstruktur und des Küstenschutzes (Art. 91a Abs. 1 GG a.F. wie n.F.) die Funktion als »Scharnier « im Rahmen einer »wechselseitigen Instrumentalisierung « zwischen der Bundesrepublik und der EG übernommen ${ }^{68}$. Ganz ähnlich wie im Bundesstaat ${ }^{69}$ führt die funktionenspezifische Kompetenzaufteilung, die Entkoppelung von Rechtsetzung und Vollzug zu Durchbrechungen von Konnexitätsansätzen, da andernfalls Politik auf Kosten »anderer « verwirklicht würde ${ }^{70}$. Der EuGH hat sich bisher nur vereinzelt zu der Frage der Verteilung der Finanzierungslast zwischen Gemeinschaft und Mitgliedstaaten geäußert. In Anwendung einer geradezu umgekehrten Konnexität schloss er von der Finanzierungsbefugnis auf die Aufgabe der Gemeinschaft ${ }^{71}$. In den Fällen zum »Internationalen Naturkautschuk-Übereinkommen « ${ }^{72}$ und zur Entwick-

66 Zur Struktur von gemeinschaftsrechtlicher Kompetenzzuweisung und -verteilung M. Nettesheim, Kompetenzen, in: von Bogdandy (Hrsg.), Europäisches Verfassungsrecht, 2003, S. 415 ff.

67 H.-C. Eiden, Die Agrarmarktordnung der Europäischen Gemeinschaften, DVBl. 1988, S. 1087 (1092); insgesamt ders., Die Vorschriften der EU zur Finanzierung der gemeinsamen Agrarpolitik, in: 65 für Albert Bleckmann, 2007, S. 109.

68 Volkmann (Fn. 7), Art. 91a Rdnr. 11; insgesamt in je unterschiedlichen Zusammenhängen H. Neupert, Regionale Strukturpolitik als Aufgabe der Länder, 1985; W. Spannowsky, Der Handlungsspielraum und die Grenzen der regionalen Wirtschaftsförderung des Bundes, 1987; S. große Siemer, Die kommunale Wirtschaftsförderung und die Regionalpolitik der Europäischen Gemeinschaften, 1993; T. Falkenkötter, Die Auswirkungen der Kohäsionspolitik der Europäischen Gemeinschaft auf die Gemeinschaftsaufgabe »Verbesserung der regionalen Wirtschaftsstruktur« nach Art. 91a Abs. 1 Nr. 2 GG, 2001.

69 W. Heun, Strukturprobleme des Finanzausgleichs, Der Staat 31 (1992), S. 205 (210): Ausnahmeregelungen in Art. 104a Abs. 2 bis 4 GG als »Konsequenz der Aufspaltung von Gesetzgebungs- und Verwaltungskompetenz«.

70 Bleckmann/Hölscheidt (Fn. 57), S. 854 f.

71 Häde (Fn. 63), S. 403.

72 EuGH, Gutachten 1/78, Slg. 1979, 2871, v.a. Rdnr. 60. 
lungspolitik ${ }^{73}$ führt er aus, es stehe Gemeinschaft und Mitgliedstaaten frei, wer die Finanzierung übernehmen wolle. Dem ist zu entnehmen, dass Finanzierungszuständigkeiten einer Seite diejenigen der anderen Seite nicht ausschließen. Wechselseitige Mitfinanzierungsmöglichkeiten sind von Gemeinschaftsrechts wegen nicht ausgeschlossen $^{74}$. Allerdings kann die Gemeinschaft die Mitgliedstaaten nicht zu bestimmten Ausgaben verpflichten. Die Einflussnahme erfolgt - wie im Bundesstaat - indirekt, mittelbar: Die Anreize, die »Ko-Finanzierungen« bieten, sind politisch nur schwer zu bändigen: »Überhaupt lautet die entscheidende Frage heute, welche Handlungsspielräume den Mitgliedsstaaten angesichts des dichten Netzes europäischer Fördermaßnahmen für ihre eigene Strukturpolitik noch verbleiben. ${ }^{75}$

\section{Verflechtung als bundesstaatliches Problem ${ }^{76}$}

Es gehört $\mathrm{zu}$ den neueren Entwicklungen der bundesstaatlichen Diskussion in Deutschland, die Probleme föderaler Verflechtung unter Topoi wie Verantwortungszusammenhang ${ }^{77}$, Intransparenz, und Individualbezug der Staatsorganisation ${ }^{78} \mathrm{zu}$ diskutieren; diese sind z.T. an die Stelle des problematischen Rekurses auf die »Eigenstaatlichkeit « der Länder getreten ${ }^{79}$. Hinter dem Neuansatz steht der Zusammenhang zwischen dem bundesstaatlichen und dem demokratischen Verfassungsprinzip. Dem liegt die Erkenntnis zugrunde, dass alle Legitimationsfragen letztlich bei der freien, autonomen Person ansetzen oder zu ihr zurückführen. Das Individuum erweist sich als letzte Bezugsgröße des Rechts. Nur von der autonomen Person aus kann Herrschaft und können institutionelle Regelungen ihre Rechtfertigung erfahren ${ }^{80}$. Dies ist im demokratischen Verfassungsstaat Folge des notwendigen Aufeinanderbezogenseins individueller und demokratischer Selbstbestimmung ${ }^{81}$ sowie individueller und demokratischer Freiheit ${ }^{82}$. Individuelle und staatliche Autonomie werden damit nicht

73 EuGH, Rs. C-316/91 (Parlament/Rat), Slg. 1994, I-625; dazu T. Henze, Aufgaben- und Ausgabenkompetenz der Europäischen Gemeinschaft und ihrer Mitgliedstaaten im Bereich der Entwicklungspolitik, EuR 1995, S. 76.

74 Häde (Fn. 63), S. 403 f.

75 Volkmann (Fn. 7), Art. 91a Rdnr. 11.

76 Der folgende Abschnitt knüpft an Waldhoff, Finanzautonomie (Fn. 56), S. 231 ff., an.

77 Hier sind v.a. die Arbeiten Peter M. Hubers zu nennen: Deutschland in der Föderalismusfalle? 2003; Gutachten D zum 65. DJT, 2004; ders. (Fn. 55), S. 22 ff., unter Hinweis auf die materielle Aufladung des demokratischen Wahlrechts aus Art. 38 GG im Maastrichturteil des Bundesverfassungsgerichts.

78 Waldhoff (Fn. 56), S. 231 ff.

79 Allgemein zur Kritik dieser Figur C. Möllers, Staat als Argument, 2000, S. 350 ff.; damit hat sich zugleich die Diskussion von einer Verengung auf den Finanzausgleich auf Grundfragen einer bundesstaatlichen Finanzverfassung gewandelt, vgl. Hellermann (Fn. 10), Rdnr. 274.

80 Im Anschluss an Kant etwa J. Habermas Faktizität und Geltung, 1992, S. 15 f., S. 122 ff.; ferner auch P. Unruh, Der Verfassungsbegriff des Grundgesetzes, 2002, S. 7 ff., 340 ff.; A. Janssen in: Henneke (Hrsg.), Verantwortungsteilung zwischen Kommunen, Ländern, Bund und EU, 2001, S. 59 (63).

81 C. Möllers Gewaltengliederung, 2006, S. 15 ff., $28 \mathrm{ff}$.

82 J. Isensee, Grundrechte und Demokratie, Der Staat 20 (1981), S. 161 ff.; C. Starck, Grundrechtliche und demokratische Freiheit, in: Isensee/Kirchhof (Hrsg.), HStR III, 3. Aufl. 2005, § 33 Rdnr. 2 und durchgehend. 
gleichgesetzt. Die wechselseitige Bezogenheit ist vielmehr Element eines Legitimationszusammenhangs zwischen Individuum und Herrschaft. Staatsorganisationsrechtliche Prinzipien und Staatsstrukturentscheidungen wurden in der deutschen Tradition demgegenüber ganz überwiegend traditional ${ }^{83}$ oder funktional ${ }^{84}$ legitimiert $^{85}$. Der Individualbezug erscheint in dieser Sichtweise eher negativ: Es wird ein Spannungsverhältnis zwischen Bundesstaatlichkeit und (parlamentarisch-)demokratischem Verfassungsprinzip konstatiert, es werden die eher gegenläufigen Wirkungen analysiert $^{86}$. Aus dem Dargelegten wird dann vielfach ein vermeintlich »unpolitischer « Charakter des Bundesstaats hergeleitet ${ }^{87}$. Der dargelegte Zusammenhang gilt für die Grundfragen der Staatsfinanzierung in besonderem Maße ${ }^{88}: »$ No taxation without representation« als Kampfbegriff der amerikanischen Revolution machte die hier zugrunde gelegte Prämisse, »dass jeder Verteilung von öffentlichen Geldern zunächst ein Zugriff auf private Gelder vorausgeht ${ }^{89}$, zum Allgemeingut. Gleichwohl verfehlten und verfehlen weite Teile der seit Existenz des deutschen Nationalstaats permanent geführten Debatte über die bundesstaatliche Finanzverfassung diesen Zusammenhang. Das Programm »No taxation without representation « ist Stufungen wie im Bundesstaat oder im Staatenverbund niemals angepasst worden. Im Rahmen der völlig parallel laufenden, weitgehend unkoordinierten Kompetenzaufteilung nach der amerikanischen bundesstaatlichen Tradition führte das auch nicht zu größeren Verwerfungen. Für die funktionale Aufteilung der Kompetenzen sowohl in der deutschen als auch in der gemeinschaftsrechtlichen Tradition gilt anderes. Die fehlende

83 J. Isensee, Der Föderalismus und der Verfassungsstaat der Gegenwart, AöR 115 (1990), S. 248 (251 f.); ders., Idee und Gestalt des Föderalismus unter dem Grundgesetz, in: ders./ Kirchhof (Hrsg.), HStR IV, 2. Aufl. 1999, § 98 Rdnr. 1, 10; M. Jestaedt, Bundesstaat als Verfassungsprinzip, in: Isensee/Kirchhof (Hrsg.), HStR II, 3. Aufl. 2004, § 29 Rdnr. 2 ff.

84 R. Mayntz, Föderalismus und die Gesellschaft der Gegenwart, AöR 115 (1990), S. 232 ff.; zur Kritik B.-O. Bryde, Auf welcher politischen Ebene sind welche Probleme vorrangig anzugehen?, in: Sitter-Liver (Hrsg.), Herausgeforderte Verfassung, 1999, S. 223 (227 f.): »funktionalistischer Kurzschluss«, indem versucht wird ein »mehrdimensionales Problem eindimensional zu lösen«; hier wäre auch die ökonomische Theorie des Bundesstaats als neuerer Ansatz einzuordnen, vgl. statt vieler D. Sauerland, Föderalismus zwischen Freiheit und Effizienz, 1997; S. Oeter, Erprobung der Konstitutionellen Politischen Ökonomie an Einzelfragen - Föderalismus, in: Engel/Morlok (Hrsg.), Öffentliches Recht als ein Gegenstand ökonomischer Forschung, 1998, S. 119 ff.

85 Diese Legitimationsmodelle können - im Gegensatz zu dem hier verfolgten - als »outputorientiert « bezeichnet werden. $\mathrm{Zu}$ dem Übergang von der traditionalen zur funktionalen Legitimation G. Kisker, Ideologische und theoretische Grundlagen der bundesstaatlichen Ordnung der Bundesrepublik Deutschland, in: Probleme des Föderalismus, 1985, S. 23 ff.

86 Einflussreich E. Kaufmann, Bismarcks Erbe in der Reichsverfassung, 1917, S. 67 ff.; $C$. Schmitt Verfassungslehre, 1928, S. 334, 388 ff.; Kontinuität dieser Ansätze in der jungen Bundesrepublik v.a. über W. Weber Spannungen und Kräfte im westdeutschen Verfassungssystem, 3. Aufl. 1970, S. 57 (63 ff.).

87 Isensee (Fn. 83), S. 258 f.

88 Vgl. etwa Diskussionsbemerkung A. Merkl, VVDStRL 14 (1956), S. 85.

89 P. Kirchhof, Diskussionsbemerkung, VVDStRL 52 (1993), S. 147 (148); im Ansatz anders F. Schoch, Rechtliche Rahmenbedingungen einer Verantwortungsteilung im Mehr-Ebenensystem, in: Henneke (Hrsg.), Verantwortungsteilung zwischen Kommunen, Ländern, Bund und EU, 2001, S. 21 (22). 
Beziehung zwischen demokratischer Partizipation an der Regelung der Staatsfinanzierung und föderaler Ordnung mag für ein Verfassungssystem, wie dasjenige des Bismarckreichs mit seinem grundlegend anders gearteten Legitimationsmodus als »Bund « souveräner Fürstenhäuser, noch nachvollziehbar sein; überhaupt standen im konstitutionellen Zeitalter Föderalismus und Bundesstaatlichkeit in Deutschland für monarchische Legitimität und Partikularismus und damit gegen die zukunftsweisenden, an das Individuum anknüpfenden Bewegungen von Nation und Demokratie ${ }^{90}$. Das stellt als historische Hypothek einen entscheidenden Legitimitätsunterschied etwa zum US-amerikanischen oder schweizerischen Föderalismus bis in die jeweilige bundesstaatliche Finanzordnung dar. Speziell für die Finanzverfassung kommen Entstehensbedingungen hinzu, wie sie für eine föderalistisch-freiheitliche Interpretation ungünstiger kaum sein könnten ${ }^{91}$. Der maßgebliche Berichterstatter im Parlamentarischen Rat, der ehemalige preußische Finanzminister Hermann Höpker Aschoff, zeichnete sich - verstärkt durch Abwehrreflexe gegen bundesstaatliche Vorgaben der alliierten Siegermächte ${ }^{92}$ - durch ein politisch, wie biographisch ${ }^{93}$ geprägtes zentralistisch-technokratisches Grundverständnis aus und wollte in Anknüpfung an Weimarer Verfassungszustände (hier vornehmlich die zentralisierende Erzbergersche Finanzreform von 1919/20 ${ }^{94}$ ) ein die föderale Gliederung letztlich negierendes Regime errichten $^{95}$. Eine demokratische Neufundierung der föderalistischen Staatsordnung konnte in einem derartigen Umfeld nicht stattfinden. Aus dieser Vorprägung hat sich die bundesstaatliche Finanzverfassung letztlich nie befreien können. In der großen Finanzreform 1967/69 wurde - wie gezeigt - die unitarisch-technokratische Grundprägung nur durch ein neues, ökonomisch inzwischen alles andere als unstrittiges Paradigma, die staatliche Konjunkturpolitik im Sinne der sog. Globalsteuerung ersetzt. Für den auf der Volkssouveränität aufbauenden und damit gerade beim autonomen Individuum anknüpfenden Verfassungsstaat kann das auch in bundesstaatlicher Hinsicht nicht das letzte Wort sein.

90 Vgl. mit Bezug auf die Finanzordnung W. Gerloff, Die Finanzgewalt im Bundesstaat, 1948, S. 9 ff.; J.-P. Schneider, Bundesstaatliche Finanzbeziehungen im Wandel, Der Staat 40 (2001), S. 272 (282); zu Bismarcks Instrumentalisierung der föderalen Reichsstruktur gegen den Parlamentarismus C. Möllers, Der parlamentarische Bundesstaat, in: Aulehner u.a. (Hrsg.), Föderalismus - Auflösung oder Zukunft der Staatlichkeit? 1997, S. 82 ff.

91 Analyse zentralistischer und unitarischer Bestrebungen im und um den Parlamentarischen Rat und die früh-bundesrepublikanische Bundesstaatslehre bei Oeter (Fn. 27), S. 96 ff., 143 ff.

92 H.J. Fischer, Parlamentarischer Rat und Finanzverfassung, Diss. iur. Kiel 1970; F.W. Scharpf, Föderale Politikverflechtung, MPIfG Working Paper 99/3, 1999, S. 4, 5.

93 T. Aders, Die Utopie vom Staat über den Parteien - Biographische Annäherungen an Hermann Höpker Aschoff (1883-1954), 1994; F. Spieker Hermann Höpker Aschoff - Vater der Finanzverfassung, 2004, insbes. S. 41 ff., 52 ff.; Waldhoff, Reformperspektiven(Fn. 31), S. 168 f.

94 Zeitgenössische Bewertung bei A. Hensel, Der Finanzausgleich im Bundesstaat, 1922, S. 174 ff.; Einzelheiten bei F. Menges Reichsreform und Finanzpolitik, 1971, S. 184 ff.

$95 \mathrm{Zu}$ diesen ausgesprochen zentralistischen Grundprägungen Gerloff (Fn. 90); H. Höpker Aschoff, Das Finanz- und Steuersystem des Bonner Grundgesetzes, AöR 75 (1949), S. 306 ff.; G. Strickrodt, Die Finanzverfassung des Bundes als politisches Problem, 1951, S. 13; W. Renzsch (Fn. 28), S. 54 ff.; Höpker Aschoffs Wirken in diese Richtung reicht bis in das erste Urteil des Bundesverfassungsgerichts zum Länderfinanzausgleich hinein (BVerfGE 1, 117), vgl. Oeter (Fn. 27), S.193 ff. 
Der ausgeprägte Individualbezug im Recht der Staatsfinanzierung in ihrer Gesamtheit erschöpft sich nach überkommener Sicht über den Vorbehalt des Gesetzes in der besonderen Gesetzesgebundenheit des Abgabenrechts. Ein Bezug zur bundesstaatlichen Finanzordnung wird nicht gezogen. Das Steuerrecht lebt - nach einer vielzitier-

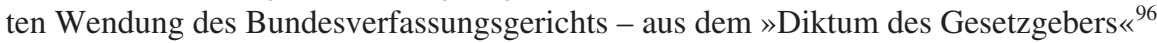
- unter dem Grundgesetz müsste man ergänzen: des Bundesgesetzgebers. Der steuerrechtliche Gesetzesvorbehalt ist nicht nur historisch Vorbild für den allgemeinen Gesetzesvorbehalt gewesen ${ }^{97}$, das Steuer- und Abgabenrecht unterliegt auch in der geltenden Verfassungsordnung einer strikten Gesetzesbindung. Im Verfassungsstaat kann sich die demokratische Fundierung der Steuer nicht in dem formalen Erfordernis der gesetzlichen Grundlage der Abgabenerhebung erschöpfen. Auch die Verwendung der erhobenen Steuern ist in spezifischer Weise demokratisch rechtfertigungsbedürftig. Dies wird - bei aller Steuerungsschwäche ${ }^{98}$ - durch das Budgetrecht des Parlaments im Sinne einer politischen Gesamtentscheidung über die Staatsausgaben gewährleistet, durch welche neben das Verwaltungsrecht als Sachrecht die Zuweisung finanzieller Mittel zur Verwirklichung materieller Verwaltungszwecke tritt ${ }^{99}$. Art. 109 Abs. 1 GG ist treffend als »finanzielle « Ausprägung des Demokratieprinzips bezeichnet worden ${ }^{100}$. Diese funktioniert jedoch nur jeweils innerhalb einer Ebene, nicht ebenenübergreifend $^{101}$.

Die so skizzierte Fundierung der bundesstaatlichen Finanzordnung verlangt die Existenz und die Funktionsfähigkeit von Verantwortlichkeit für politische Entscheidungen und damit auch für die Verausgabung von Finanzmitteln. Solche Verantwortungszusammenhänge bauen in der Demokratie auf der Zurechenbarkeit von Verantwortung auf ${ }^{102}$. Um die demokratische Rückkopplung sicherzustellen, müssen diese Verantwortlichkeiten dafür im Sinne einer Verantwortungsklarheit auch sichtbar

96 BVerfGE 13, 318 (328) im Anschluss an O. Bühler/G. Strickrodt, Steuerrecht, Bd. 1, 3. Aufl. 1960, S. 658.

97 E.-W. Böckenförde, Gesetz und gesetzgebende Gewalt, 2. Aufl. 1981, 47 ff.; H.-J. Papier, Die finanzrechtlichen Gesetzesvorbehalte und das grundgesetzliche Demokratieprinzip, 1973, S. $15 \mathrm{ff}$.

98 Vgl. nur W. Heun, Staatshaushalt und Staatsleitung, 1989; C. Gröpl, Haushaltsrecht und Reform, 2001, S. 152 ff., 165 und öfter.

99 P. Kirchhof, Die Steuerung des Verwaltungshandelns durch Haushaltsrecht und Haushaltskontrolle, NVwZ 1983, S. 505 ff.; Heun, Staatshaushalt (Fn. 98), S. 270 ff.; C. Waldhoff, Die Zwecksteuer, StuW 2002, S. 285 (299).

100 F. Kirchhof, Empfehlen sich Maßnahmen, um in der Finanzverfassung Aufgaben- und Ausgabenverantwortung von Bund, Ländern und Gemeinden stärker zusammenzuführen?, Gutachten zum 61. DJT, 1996, Rdnr. D 53 Fn. 215.

101 Heintzen (Fn. 60), Vorb. Art. 104a-115 Rdnr. 46; C. Hillgruber, Klarere Verantwortungsteilung von Bund, Ländern und Gemeinden?, JZ 2004, S. 837 (845); von seinem Ausgangspunkt, die demokratische und die föderale Frage getrennt zu denken, ist dies für Schoch (Fn. 89), S. 24, folgerichtig.

102 H.-J. Vogel, Die bundesstaatliche Ordnung des Grundgesetzes, in: Benda/Maihofer/Vogel (Hrsg.); Handbuch des Verfassungsrechts, 2. Aufl. 1994, § 22 Rdnr. 13; Kisker (Fn. 85), S. 34; Lerche (Fn. 7), Art. 83 Rdnr. 107, 110; U. Volkmann, Bundesstaat in der Krise?, DÖV 1998, S. 613 (615). 
$\operatorname{sein}^{103}$. Auf die Staatsfinanzierung bezogen ist dies nicht nur ein finanzpsychologisches Postulat zur Vermeidung von Abgabenwiderstand und damit zur Hebung der Steuermoral, sondern essentieller Bestandteil demokratischer Legitimationsstiftung. Durch die der deutschen Bundesstaatstradition entsprechende, funktionenspezifische Aufteilung der Finanzhoheiten hinsichtlich der einzelnen Einnahmen bei ebenenangesiedelter Budgetverantwortung fehlt ein entscheidendes Bindeglied in der Verantwortungsklarheit, der Zurechenbarkeit von Verantwortung und damit demokratischer Rückkopplung im Bereich des Finanzwesens ${ }^{104}$. Historisch vorbelastet harren somit die demokratischen Verantwortungszusammenhänge als Argumentationsreserve in der Anwendung der geltenden Finanzverfassung und als Leitgesichtspunkt de constitutione ferenda im bundesstaatlichen Finanzbereich der Freilegung ${ }^{105}$.

\section{Die Mischfinanzierung in der Föderalismusreform II}

Für die Mischfinanzierungen - ihre Analyse de constitutione lata wie für Reformoptionen de constitutione ferenda - sind vor allem zwei Aspekte herauszustellen: Die Funktion der Finanzierungskompetenz vor dem Hintergrund notwendiger demokratischer Verantwortungszusammenhänge sowie die Interdependenz der Finanzierungsverantwortung mit den anderen finanzverfassungsrechtlichen (Teil-)Hoheiten, letztlich mit der bundesstaatlichen Aufgabenverteilung insgesamt. Ein unbefangener Beobachter könnte erstaunt sein, sähe er den Aufwand der Diskussion um Art. 91a f.; 104a f. GG, das Konnexitätsprinzip und die dieses durchbrechenden Mischfinanzie-

103 Damit wird der einen Kern des Demokratieprinzips darstellende Zusammenhang der Koppelung von Politik, öffentlicher Meinung und individuellem Bewusstsein durch ein Sichtbarmachen politischen Entscheidens verwirklicht, vgl. nur Udo Di Fabio, Das Recht offener Staaten, 1998, S. 45 ff.; in bundesstaatlichem Kontext Oeter (Fn. 27), 1998, S. 581 f.

104 P. Kirchhof, Der Verfassungsauftrag zum Länderfinanzausgleich als Ergänzung fehlender und als Garant vorhandener Finanzautonomie, 1982, S. 14; zur insofern begrenzten Bedeutung von Art. 109 Abs. 1 GG bereits BVerfGE 1, 117 (131).

105 Entsprechende Kritik an den Gemeinschaftsaufgaben einschließlich der damit verbundenen Mischfinanzierungstatbestände bereits bei Soell (Fn. 5), S. 402 ff. Treffend spricht P. Selmer, Empfehlen sich Maßnahmen, um in der Finanzverfassung Aufgaben- und Ausgabenverantwortung von Bund, Ländern und Gemeinden stärker zusammenzuführen? NJW 1996, S. 2062, von einem »Postulat demokratischer Finanzverantwortung«. Die Mahnung, bei dem Projekt einer Stärkung der Landes-(finanz-)autonomie dürfe es sich nicht um einen ahistorischen Aufbruch »zu den Quellen des deutschen Staatsorganisationsrechts « handeln und der Gefahr »föderaler Romantik « müsse gegengesteuert werden, daher sei eine »traditionsbewusste Reformulierung « anzustreben (J.-P. Schneider, Bundesstaatliche Finanzbeziehungen im Wandel, Der Staat 40 (2001), S. 272 (284)) bzw. die Kompatibilität mit der »inneren Rationalität«, die »Vereinbarkeit mit der deutschen bundesstaatlichen Kultur « sei zu berücksichtigen (Stefan Korioth, Klarere Verantwortungsteilung von Bund, Ländern und Kommunen in der Finanzverfassung?, Referat zum 65. DJT 2004, Rdnr. P 89 (90, 97 ff.); ders. (Fn. 4), S. 21)), darf nicht dazu führen, machtstaatlich-unitarische Tendenzen der deutschen Bundesstaatstradition zu transportieren; insofern geht es bei dem hier verfolgten Anliegen weniger um die Rekonstruktion von Verlusten, als um die Neujustierung in einer grundlegend anders legitimierten Verfassungsordnung. 
rungen, denn »es versteht sich ja von selbst, dass in einem Bundesstaat jede Ebene diejenigen Ausgaben zu tragen hat, die bei Wahrnehmung ihrer Aufgaben anfallen « ${ }^{106}$. Angesichts des Faktums, dass jede Ausgabe letztlich von den steuerzahlenden Bürgern finanziert wird, müssen Entscheidungsverantwortung und Ausgabenlast grundsätzlich zusammenfallen. Ausgangspunkt allen bundesstaatlichen Argumentierens ist es dabei, dass dieser »zuallererst auf Trennung der Kompetenzen und Bewahrung der Selbständigkeit des Zentralstaats wie der Länder angelegt « ist ${ }^{107}$. Dieser auch genuin-demokratische Zusammenhang wird durch die dem GG eigentümliche Aufspaltung zwischen Gesetzgebung und Vollzug komplex ${ }^{108}$ : Im Vollzugsföderalismus deutscher Prägung ist $\mathrm{zu}$ entscheiden, ob »Aufgabe « in Art. 104a Abs. 1 GG auf die Gesetzgebung oder auf die Vollziehung bezogen ist ${ }^{109}$. Letztlich besteht hier ein Dilemma ${ }^{110}$ : Entweder wird die Verwaltung oder der Gesetzgeber von den Kostenfolgen ihres Handelns teilweise freigestellt. Die traditionelle Bezugnahme der Konnexität auf die Verwaltungszuständigkeit ${ }^{111}$ überzeugt auch heute noch, sofern Sicherungsmechanismen vorhanden sind, welche die Abwälzung der Finanzierungslast auf eine andere Ebene verhindern oder erschweren ${ }^{112}$. Das sind zum einen die Durchbrechungen des Art. 104a Abs. 2 und 3 GG (alter wie neuer Fassung), die zu Mischfinanzierungen führen (können), zum anderen ist es der neue Abs. 4 dieser Norm, der einer Ländermehrheit über den Bundesrat die Möglichkeit gibt, entsprechende Belastungen abzuwehren bzw. Kompensationen auszuhandeln. Hier ist zwar in der Tat ein Zielkonflikt mit dem Postulat der Zurückdrängung der Zustimmungstatbestände nicht zu leugnen ${ }^{113}$. Gleichwohl bewirkt diese »Verflechtung « im Bereich der Finanzierungslast mittelbar ein Stück »Entflechtung«. Für die kommunale Ebene sind die in ihrer Bedeutung kaum zu überschätzenden Art. 84 Abs. 1 Satz 7; 85 Abs. 1 Satz 2 GG n.F. anzuführen. Der verfassungspolitisch lange geforderte Übergang zu der Alternative einer Gesetzeskausalität der Konnexität ist damit weitgehend hinfällig geworden; er würde zudem die Länder entscheidend schwächen, gerieten sie doch in neue Abhängigkeiten vom Bund und mutierten sie ohne eigene umfassende Finanzausstattung zu Zuweisungsempfängern des Bundes ${ }^{114}$.

106 von Arnim (Fn. 19), Rdnr. 11; H.J. Blanke, Die Finanzverfassung als Spiegelbild föderaler Kompetenzverteilung, in: ders./Schwanengel (Hrsg.), Zustand und Perspektiven des deutschen Bundesstaates, 2005, S. 127 (132).

107 Heun (Fn. 8), Art. 91a Rdnr. 1.

108 W. Heun, Strukturprobleme des Finanzausgleichs, Der Staat 31 (1992), S. 205 (209 f.); Hellermann (Fn. 8), Art. 104a Rdnr. 14 f.

109 Zur Diskussion über einen Übergang von der Vollzugs- zur Gesetzeskausalität vgl. nur Henneke (Fn. 2), Rdnr. 212 ff.; Prokisch (Fn. 8), Rdnr. 91 ff.

110 Müller-Volbehr (Fn. 38), S. 318, spricht von »Antinomien«, welche das Problem zur »Ewigkeitsfrage jeder Reformdiskussion« machten.

111 Vgl. nur Prokisch (Fn. 8), Rdnr. 85 ff.

112 Vgl. auch Selmer (Fn. 105), S. 2062 ff.; Heun (Fn. 8), Art. 104a Rdnr. 12; ders. (Fn. 3), S. $1020 \mathrm{ff}$.

113 Korioth (Fn. 4), S. 3 ff.

114 Selmer (Fn. 17), S. 874 f.; Huber, Bund-Länder-Verhältnis (Fn. 55), S. 43). 
Angesichts der dargelegten Verantwortungszusammenhänge ist das zu Mischfinanzierungen führende Übergreifen der einen Ebene auf die andere zu bewerten, denn die Finanzierungskompetenz bietet auch Möglichkeiten der Einflussnahme, der Steuerung durch das Medium Geld ${ }^{115}$. Wie die lange Diskussion um Fondsverwaltung und Investitionshilfekompetenzen zeigt, handelt es sich dabei zwar um ein subtiles, da indirekt steuerndes, gleichwohl um so wirkungsvolleres Mittel der Einflussnahme. Durch solche Einflussnahme wird nicht nur die bundesstaatliche Kompetenzordnung, sondern auch der demokratische Verantwortungszusammenhang gestört, sie ist daher in hohem Maße rechtfertigungsbedürftig. Ausnahmetatbestände in der Verfassung sind - wie das Bundesverfassungsgericht treffend herausgearbeitet hat - entsprechend eng und vorsichtig auszulegen und anzuwenden ${ }^{116}$.

Demgegenüber ist das Festhalten an den Gemeinschaftsaufgaben verfehlt ${ }^{117}$. Hier ist die Föderalismusreform - auch jenseits von Bildern eines »idealisierten Bundesstaates $\ll^{118}$ - in der Tat halbherzig und inkonsequent geblieben. Jenseits der Mischfinanzierung wirft die durch die Gemeinschaftsaufgaben angeordnete oder ermöglichte Mischverwaltung zusätzliche Probleme auf. Eine Streichung, die auch die Mitfinanzierungstatbestände beträfe, müsste allerdings den Ländern entsprechende Finanzmittel zuweisen ${ }^{119}$. Als kompensatorisches Steuerungselement für den Bund könnte Art. $104 \mathrm{~b}$ GG dienen ${ }^{120}$. Gewisse Korrekturmechanismen einer strengen Vollzugskausalität und damit Mischfinanzierungen sind für den praktisch arbeitenden Bundesstaat notwendig ${ }^{121}$. Dies stellt sich nicht zuletzt als Folge der Interdependenz der Frage der Finanzierungskompetenz mit der allgemeinen bundesstaatlichen und finanzverfassungsrechtlichen Zuständigkeitsordnung dar ${ }^{122}$. Das gilt für die einem verfehlten Modell nachhängenden Gemeinschaftsaufgaben jedoch keinesfalls ${ }^{123}$. Die Gemeinsame Kommission zur Modernisierung der Bund-Länder-Finanzbeziehungen sollte - allen defensiven Äußerungen zum Trotz - den Mut und die Kraft aufbringen, bei ihren Überlegungen auch die bisher unvollständige und teilweise missglückte Reform der Finanzierungskompetenz und damit der Mischfinanzierung erneut zu überdenken.

115 Dazu umfassend K. Vogel, Der Finanz- und Steuerstaat, in: Isensee/Kirchhof (Hrsg.), HStR II, 3. Aufl. 2004, § 30 Rdnr. 22 ff.; auf Mischfinanzierungen bezogen bereits früh Soell (Fn. 5).

116 BVerfGE 39, 95 (107 ff.); 41, 291 (304 ff.).

117 Selmer (Fn. 17), S. 874; für den Hochschulbereich früh bereits C. Flämig, Stand und Programmatik der Bildungsfinanzierung, WissR 14 (1981), S. 112 (129).

118 Heun (Fn. 8), Art. 91a Rdnr. 8.

119 Wendt (Fn. 36), Rdnr. 94; Merten (Fn. 41), S. 85.

120 Bezogen auf Art. 104a Abs. 4 GG a.F. Schmidt-Jortzig (Fn. 41), S. 26 f.; Huber, Bund-Länder-Verhältnis (Fn. 55), S. $44 \mathrm{ff}$.

121 Heun, Strukturprobleme (Fn. 108), S. 210; Korioth (Fn. 4), S. 6.

122 Hellermann (Fn. 8), Art. 104a Rdnr. 7 ff.

123 A.A. Heun (Fn. 8), Art. 91a Rdnr. 8. 